Indian J. Pure Appl. Math., 51(2): 717-733, June 2020

(c) Indian National Science Academy

DOI: $10.1007 / \mathrm{s} 13226-020-0426-4$

\title{
ARITHMETIC PROPERTIES FOR 7-REGULAR PARTITION TRIPLES
}

\author{
Shane Chern*, Dazhao Tang** and Ernest X. W. Xia*** \\ *Department of Mathematics, The Pennsylvania State University, \\ University Park, PA 16802, USA \\ ** College of Mathematics and Statistics, Chongqing University, \\ Huxi Campus LD206, Chongqing 401331, P.R. China \\ ${ }^{* * *}$ Department of Mathematics, Jiangsu University, Zhenjiang, \\ Jiangsu, 212013, P.R. China \\ e-mails: shanechern@psu.edu; chenxiaohang92@gmail.com; \\ dazhaotang@sina.com; Ernestxwxia@163.com \\ (Received 17 November 2018; after final revision 23 January 2019; \\ accepted 5 April 2019)
}

Let $T_{\ell}(n)$ denote the number of $\ell$-regular partition triples of $n$. In this paper, we consider the arithmetic properties of $T_{7}(n)$. An infinite family of congruences modulo powers of 7 and several congruences modulo 7 are established. For instance, we prove that for all $n \geq 0$ and $\alpha \geq 1$,

$$
T_{7}\left(7^{2 \alpha} n+\frac{3 \times 7^{2 \alpha}-3}{4}\right) \equiv 0 \quad\left(\bmod 7^{\alpha}\right) .
$$

Key words : Partitions; arithmetic properties; $\ell$-regular partition triples.

2010 Mathematics Subject Classification : 05A17, 11P83.

\section{INTRODUCTION}

A partition [3] of a positive integer $n$ is a finite weakly decreasing sequence of positive integers $\pi_{1} \geq \pi_{2} \geq \cdots \geq \pi_{r}$ whose sum equals $n$. The $\pi_{i}$ 's are called the parts of this partition. We denote by $p(n)$ the number of partitions of $n$, and adopt the convention that $p(0)=1$. The generating function of $p(n)$ satisfies the identity

$$
\sum_{n=0}^{\infty} p(n) q^{n}=\frac{1}{(q ; q)_{\infty}}
$$


where we use the customary $q$-series notation

$$
(a ; q)_{\infty}:=\prod_{n=0}^{\infty}\left(1-a q^{n}\right) .
$$

An $\ell$-regular partition is a partition where none of the parts are divisible by $\ell$. If $b_{\ell}(n)$ denotes the number of $\ell$-regular partitions of $n$, then its generating function satisfies the identity

$$
\sum_{n=0}^{\infty} b_{\ell}(n) q^{n}=\frac{\left(q^{\ell} ; q^{\ell}\right)_{\infty}}{(q ; q)_{\infty}} .
$$

The arithmetic properties of $b_{\ell}(n)$ have been studied by a number of mathematicians (see, for instance, [7, 8, 10, 11, 17-21, 28-31]). Recently, Baruah and Das [5] established several congruences modulo 2 and 7 for $b_{7}(n)$. We also remark that Dandurand and Penniston indeed gave exact criteria for when $b_{7}(n)$ is divisible by 7 in terms of the prime factorization of $4 n+1$ in a prior paper [12]. Further, Ahmed and Baruah [2] established several congruences for $b_{\ell}(n)$ with $\ell \in\{5,6,7,49\}$, showing for example that

$$
b_{7}\left(7^{3} \times 3^{2 \alpha+2} n+\frac{7^{3} \times 5 \times 3^{2 \alpha+1}-1}{4}\right) \equiv 0 \quad(\bmod 147)
$$

and

$$
b_{7}\left(7^{3} \times 3^{2 \alpha+3} n+\frac{7^{3} \times 11 \times 3^{2 \alpha+2}-1}{4}\right) \equiv 0 \quad(\bmod 147) .
$$

Quite recently, Adiga and Ranganatha [1, Theorem 1.1] as well as Wang [26, Theorem 1.1] independently proved the following infinite family of congruences modulo powers of 7 for $b_{7}(n)$ :

$$
b_{7}\left(7^{2 \alpha-1} n+\frac{3 \times 7^{2 \alpha-1}-1}{4}\right) \equiv 0 \quad\left(\bmod 7^{\alpha}\right) .
$$

The interested reader may also refer to [22, 24-26] for other congruences modulo arbitrary powers of $\ell$ for $\ell$-regular partitions or partition pairs where $\ell$ is a small prime.

Let $T_{\ell}(n)$ denote the number of $\ell$-regular partition triples of $n$, whose generating function satisfies the identity

$$
\sum_{n=0}^{\infty} T_{\ell}(n) q^{n}=\frac{\left(q^{\ell} ; q^{\ell}\right)_{\infty}^{3}}{(q ; q)_{\infty}^{3}}
$$

Saikia and Boruah [23] considered arithmetic properties of $T_{\ell}(n)$ for $2 \leq \ell \leq 5$. In this paper, we study the arithmetic properties of $T_{7}(n)$. Our main results can be stated as follows. 
Theorem 1.1 - For all $n \geq 0$ and $\alpha \geq 1$, we have

$$
T_{7}\left(7^{2 \alpha} n+\frac{3 \times 7^{2 \alpha}-3}{4}\right) \equiv 0 \quad\left(\bmod 7^{\alpha}\right) .
$$

Theorem 1.2 - For all $n \geq 0$, we have

$$
T_{7}(7 n+1) \equiv 3 b_{7}(n) \quad(\bmod 7)
$$

Remark 1.3 : Recalling the criteria for when $b_{7}(n)$ is divisible by 7 established by Dandurand and Penniston [12], one may obtain endless families of congruences modulo 7 for $T_{7}(n)$ from Theorem 1.2 .

Theorem 1.4 - For all $n \geq 0$ and $\alpha \geq 1$, we have

$$
T_{7}(25 n+18) \equiv T_{7}(5 n+3)+3 T_{7}(n) \quad(\bmod 7)
$$

and

$$
T_{7}\left(5^{4 \alpha} n+\frac{3 \times 5^{4 \alpha}-3}{4}\right) \equiv 5^{\alpha} T_{7}(n) \quad(\bmod 7)
$$

Theorem 1.5 - For all $n \geq 0$ and $\alpha \geq 1$, we have

$$
T_{7}(169 n+126) \equiv 3 T_{7}(13 n+9)+6 T_{7}(n) \quad(\bmod 7)
$$

and

$$
T_{7}\left(13^{4 \alpha} n+\frac{3 \times 13^{4 \alpha}-3}{4}\right) \equiv(-1)^{\alpha} T_{7}(n) \quad(\bmod 7)
$$

Theorem 1.6 - For all $n \geq 0$, we have

$$
T_{7}(3 n+2) \equiv 0 \quad(\bmod 9) .
$$

\section{PRELiminary Results}

To prove the main results of this paper, we start by introducing some necessary lemmas. Here we adopt the notation

$$
E_{j}:=\left(q^{j} ; q^{j}\right)_{\infty}
$$


Let

$$
R:=R\left(q^{5}\right)=\frac{\left(q^{5} ; q^{25}\right)_{\infty}\left(q^{20} ; q^{25}\right)_{\infty}}{\left(q^{10} ; q^{25}\right)_{\infty}\left(q^{15} ; q^{25}\right)_{\infty}}
$$

Watson [27] used the quintuple product identity [9] to prove that

$$
\frac{E_{1}}{E_{25}}=\frac{1}{R}-q-q^{2} R
$$

Further, Hirschhorn [14] established the identity

$$
\frac{E_{5}^{6}}{E_{25}^{6}}=\frac{1}{R^{5}}-11 q^{5}-q^{10} R^{5}
$$

Following Hirschhorn [15], we introduce a "huffing" operator $H_{k}$, given by

$$
H_{k}\left(\sum_{n=0}^{\infty} a_{n} q^{n}\right):=\sum_{n=0}^{\infty} a_{k n} q^{k n} .
$$

As in Garvan [13], we define an infinite matrix $\{m(i, j)\}_{i, j \geq 1}$ as follows. The first seven rows are

$$
\begin{aligned}
& \left(7,7^{2}, 0,0, \ldots\right) \\
& \left(10,9 \times 7^{2}, 2 \times 7^{4}, 7^{5}, 0,0, \ldots\right) \\
& \left(3,114 \times 7,85 \times 7^{3}, 24 \times 7^{5}, 3 \times 7^{7}, 7^{8}, 0,0, \ldots\right) \\
& \left(0,82 \times 7,176 \times 7^{3}, 845 \times 7^{4}, 272 \times 7^{6}, 46 \times 7^{8}, 4 \times 7^{10}, 7^{11}, 0,0, \ldots\right), \\
& \left(0,190,1265 \times 7^{2}, 1895 \times 7^{4}, 1233 \times 7^{6}, 3025 \times 7^{7}, 620 \times 7^{9}, 75 \times 7^{11},\right. \\
& \left.\quad 5 \times 7^{13}, 7^{14}, 0,0, \ldots\right), \\
& \left(0,27,736 \times 7^{2}, 16782 \times 7^{3}, 20424 \times 7^{5}, 12825 \times 7^{7}, 4770 \times 7^{9}, 7830 \times 7^{10},\right. \\
& \left.\quad 1178 \times 7^{12}, 111 \times 7^{14}, 6 \times 7^{16}, 7^{17}, 0,0 \ldots\right), \\
& \left(0,1,253 \times 7^{2}, 1902 \times 7^{4}, 4246 \times 7^{6}, 31540 \times 7^{7}, 19302 \times 7^{9}, 7501 \times 7^{11},\right. \\
& \left.\quad 1944 \times 7^{13}, 2397 \times 7^{14}, 285 \times 7^{16}, 22 \times 7^{18}, 7^{20}, 7^{20}, 0,0, \ldots\right) .
\end{aligned}
$$

Next, $m(i, 1)=0$ for $i \geq 4$ and $m(i, 2)=0$ for $i \geq 8$. Finally, for $i \geq 8$ and $j \geq 3$, we define $m(i, j)$ recursively by

$$
\begin{aligned}
m(i, j)= & 49 m(i-1, j-1)+35 m(i-2, j-1)+7 m(i-3, j-1) \\
& +343 m(i-1, j-2)+343 m(i-2, j-2)+147 m(i-3, j-2) \\
& +49 m(i-4, j-2)+21 m(i-5, j-2)+7 m(i-6, j-2) \\
& +m(i-7, j-2) .
\end{aligned}
$$


An easy induction argument yields the following result.

Lemma 2.1 - We have

(1) $m(i, j)=0$ for $j \geq 2 i+1$.

(2) $m(i, j)=0$ for $i \geq 4 j$.

The following lemma is the main ingredient for our proof.

Lemma 2.2 — [Lemma 3.1, [13]]. Let

$$
\zeta=\frac{E_{1}}{q^{2} E_{49}} \quad \text { and } \quad T=\frac{E_{7}^{4}}{q^{7} E_{49}^{4}} .
$$

Then for all $i \geq 1$, we have

$$
H_{7}\left(\frac{1}{\zeta^{i}}\right)=\sum_{j=1}^{\infty} \frac{m(i, j)}{T^{j}}=\sum_{j=1}^{2 i} \frac{m(i, j)}{T^{j}} .
$$

We also require the following results stated by Hirschhorn [16]. Denoting

$$
\eta=\frac{E_{1}}{q^{7} E_{169}} \quad \text { and } \quad S=\frac{E_{13}^{2}}{q^{13} E_{169}^{2}},
$$

we have

$$
\begin{aligned}
H_{13}(\eta)= & \\
H_{13}\left(\eta^{2}\right)= & -2 S-1 \\
H_{13}\left(\eta^{3}\right)= & 13 \\
H_{13}\left(\eta^{4}\right)= & 2 S^{2}-13 \\
H_{13}\left(\eta^{5}\right)= & -20 S^{2}-10 \times 13 S-13^{2} \\
H_{13}\left(\eta^{6}\right)= & 10 S^{3}-13^{2} \\
H_{13}\left(\eta^{7}\right)= & 98 S^{3}+28 \times 13 S^{2}-13^{3} \\
H_{13}\left(\eta^{8}\right)= & -70 S^{4}-13^{3} \\
H_{13}\left(\eta^{9}\right)= & -162 S^{4}+108 \times 13 S^{3}+72 \times 13^{2} S^{2}+18 \times 13^{3} S+13^{4}, \\
H_{13}\left(\eta^{10}\right)= & 238 S^{5}-13^{4} \\
H_{13}\left(\eta^{11}\right)= & -902 S^{5}-1672 \times 13 S^{4}-792 \times 13^{2} S^{3} \\
& -198 \times 13^{3} S^{2}-22 \times 13^{4} S-13^{5} \\
H_{13}\left(\eta^{12}\right)= & -418 S^{6}-13^{5} .
\end{aligned}
$$


Finally, the modular equation of thirteenth order can be stated as follows.

Lemma 2.3 - [Eq. (6.40), [16]]. We have

$$
\begin{aligned}
\eta^{13} & -13 \eta^{12}+13(S+7) \eta^{11}-13^{2}(S+3) \eta^{10}+13\left(6 S^{2}+91 S+195\right) \eta^{9} \\
& -13\left(74 S^{2}+481 S+845\right) \eta^{8}+13\left(20 S^{3}+494 S^{2}+13^{3} S+3211\right) \eta^{7} \\
& -13\left(222 S^{3}+2392 S^{2}+8619 S+10985\right) \eta^{6} \\
& +13\left(38 S^{4}+1326 S^{3}+9464 S^{2}+13^{4} S+32955\right) \eta^{5} \\
& -13\left(346 S^{4}+5486 S^{3}+31096 S^{2}+81289 S+85683\right) \eta^{4} \\
& +13\left(36 S^{5}+1638 S^{4}+17238 S^{3}+83486 S^{2}+199927 S+199927\right) \eta^{3} \\
& -13\left(204 S^{5}+4498 S^{4}+37518 S^{3}+162578 S^{2}+13^{5} S+13^{5}\right) \eta^{2} \\
& +13\left(11 S^{6}+468 S^{5}+6422 S^{4}+43940 S^{3}+171366 S^{2}+13^{5} S+13^{5}\right) \eta \\
& -S^{7}=0 .
\end{aligned}
$$

\section{Proof OF THEOREM 1.1}

We first define an infinite matrix $\{a(j, k)\}_{j, k \geq 1}$ whose first row is

$$
\left(3,114 \times 7,85 \times 7^{3}, 24 \times 7^{5}, 3 \times 7^{7}, 7^{8}, 0,0, \ldots\right)
$$

and which satisfies

$$
a(j, k)= \begin{cases}\sum_{i=1}^{\infty} a(j-1, i) m(4 i-1, i+k-1) & \text { if } j \text { is odd } \\ \sum_{i=1}^{\infty} a(j-1, i) m(4 i-3, i+k-1) & \text { if } j \text { is even }\end{cases}
$$

for $j \geq 2$ and $k \geq 1$.

Remark 3.1 : It is worth pointing out that in each row of $\{a(j, k)\}_{j, k \geq 1}$, there are only finite many nonzero elements. This can be shown easily by induction on $j$. We first notice that the $j=1$ case is ensured by the construction. When $j$ is even, we see from Lemma 2.1 that $m(4 i-3, i+k-1)=0$ provided that $i+k-1 \geq 2(4 i-3)+1$, namely, $i \leq(k+4) / 7$. Further, $a(j-1, i)=0$ for sufficiently large $i$ by the inductive assumption. Hence, we can ensure that at least one of the $m(4 i-3, i+k-1)$ and $a(j-1, i)$ is 0 for all $i \geq 1$ when $k$ is large enough. When $j$ is odd, we may use a similar argument. Consequently, the summations in (3.1) are finite. 
Theorem 3.2 - For all $j \geq 1$, we have

$$
\sum_{n=0}^{\infty} T_{7}\left(7^{2 j-1} n+\frac{7^{2 j-1}-3}{4}\right) q^{n}=\sum_{\ell=1}^{\infty} a(2 j-1, \ell) q^{\ell-1}\left(\frac{E_{7}}{E_{1}}\right)^{4 \ell-3}
$$

and

$$
\sum_{n=0}^{\infty} T_{7}\left(7^{2 j} n+\frac{3 \times 7^{2 j}-3}{4}\right) q^{n}=\sum_{\ell=1}^{\infty} a(2 j, \ell) q^{\ell-1}\left(\frac{E_{7}}{E_{1}}\right)^{4 \ell-1}
$$

PROOF : By the definition of $\zeta$, we see that

$$
\sum_{n=0}^{\infty} T_{7}(n) q^{n+6}=q^{6} \frac{E_{7}^{3}}{E_{1}^{3}}=\frac{E_{7}^{3}}{E_{49}^{3}} \zeta^{-3}
$$

Applying the operator $H_{7}$ to both sides of (3.4) and using Lemma 2.2, we find that, after simplification,

$$
\begin{aligned}
\sum_{n=0}^{\infty} T_{7}(7 n+1) q^{n+2}= & q \frac{E_{1}^{3}}{E_{7}^{3}} \sum_{j=1}^{6} m(3, j) \times\left(\frac{q E_{7}^{4}}{E_{1}^{4}}\right)^{j} \\
= & 3 q^{2} \frac{E_{7}}{E_{1}}+114 \times 7 q^{3} \frac{E_{7}^{5}}{E_{1}^{5}}+85 \times 7^{3} q^{4} \frac{E_{7}^{9}}{E_{1}^{9}}+24 \times 7^{5} q^{5} \frac{E_{7}^{13}}{E_{1}^{13}} \\
& +3 \times 7^{7} q^{6} \frac{E_{7}^{17}}{E_{1}^{17}}+7^{8} q^{7} \frac{E_{7}^{21}}{E_{1}^{21}} .
\end{aligned}
$$

Dividing both sides by $q^{2}$, we see that (3.2) holds for $j=1$.

We next assume that (3.2) is true for some $j \geq 1$. Notice that (3.2) can be restated as

$$
\sum_{n=0}^{\infty} T_{7}\left(7^{2 j-1} n+\frac{7^{2 j-1}-3}{4}\right) q^{n-5}=\frac{E_{49}^{3}}{E_{7}^{3}} \sum_{\ell=1}^{\infty} a(2 j-1, \ell) T^{\ell} \zeta^{-(4 \ell-3)} .
$$

Applying the operator $H_{7}$ to both sides of the above identity and then utilizing (2.4), we deduce that

$$
\begin{aligned}
\sum_{n=0}^{\infty} T_{7} & \left(7^{2 j-1}(7 n+5)+\frac{7^{2 j-1}-3}{4}\right) q^{7 n} \\
& =\frac{E_{49}^{3}}{E_{7}^{3}} \sum_{\ell=1}^{\infty} a(2 j-1, \ell) T^{\ell} H_{7}\left(\zeta^{-(4 \ell-3)}\right) \\
& =\frac{E_{49}^{3}}{E_{7}^{3}} \sum_{\ell=1}^{\infty} \sum_{k=1}^{\infty} a(2 j-1, \ell) m(4 \ell-3, k) T^{\ell-k}
\end{aligned}
$$


From Lemma 2.1, we know that $m(4 \ell-3, k)=0$ when $1 \leq k<\ell$. It follows by replacing $q^{7}$ with $q$ that

$$
\begin{aligned}
\sum_{n=0}^{\infty} T_{7} & \left(7^{2 j} n+\frac{3 \times 7^{2 j}-3}{4}\right) q^{n} \\
& =\frac{E_{7}^{3}}{E_{1}^{3}} \sum_{\ell=1}^{\infty} \sum_{k=\ell}^{\infty} a(2 j-1, \ell) m(4 \ell-3, k)\left(q \frac{E_{7}^{4}}{E_{1}^{4}}\right)^{k-\ell} \\
& =\frac{E_{7}^{3}}{E_{1}^{3}} \sum_{\ell=1}^{\infty} \sum_{k=\ell}^{\infty} a(2 j-1, \ell) m(4 \ell-3, k+\ell-1)\left(q \frac{E_{7}^{4}}{E_{1}^{4}}\right)^{k-\ell} \\
& =\sum_{k=1}^{\infty} \sum_{\ell=1}^{\infty} a(2 j-1, \ell) m(4 \ell-3, k+\ell-1) q^{k-1}\left(\frac{E_{7}}{E_{1}}\right)^{4 k-1} \\
& =\sum_{k=1}^{\infty} a(2 j, k) q^{k-1}\left(\frac{E_{7}}{E_{1}}\right)^{4 k-1} .
\end{aligned}
$$

This implies that (3.3) holds for $j$.

We further rewrite the above identity as

$$
\sum_{n=0}^{\infty} T_{7}\left(7^{2 j} n+\frac{3 \times 7^{2 j}-3}{4}\right) q^{n-1}=\frac{E_{49}}{E_{7}} \sum_{\ell=1}^{\infty} a(2 j, \ell) T^{\ell} \zeta^{-(4 \ell-1)}
$$

Applying the operator $H_{7}$ to both sides of the above identity yields

$$
\begin{aligned}
\sum_{n=0}^{\infty} T_{7} & \left(7^{2 j}(7 n+1)+\frac{3 \times 7^{2 j}-3}{4}\right) q^{7 n} \\
& =\frac{E_{49}}{E_{7}} \sum_{\ell=1}^{\infty} a(2 j, \ell) T^{\ell} H_{7}\left(\zeta^{-(4 \ell-1)}\right) \\
& =\frac{E_{49}}{E_{7}} \sum_{\ell=1}^{\infty} \sum_{k=1}^{\infty} a(2 j, \ell) m(4 \ell-1, k) T^{\ell-k}
\end{aligned}
$$

From Lemma 2.1, we know that $m(4 \ell-1, k)=0$ when $1 \leq k<\ell$. Replacing $q^{7}$ with $q$, we have

$$
\begin{aligned}
\sum_{n=0}^{\infty} T_{7} & \left(7^{2 j+1} n+\frac{7^{2 j+1}-3}{4}\right) q^{n} \\
& =\frac{E_{7}}{E_{1}} \sum_{\ell=1}^{\infty} \sum_{k=\ell}^{\infty} a(2 j, \ell) m(4 \ell-1, k)\left(q \frac{E_{7}^{4}}{E_{1}^{4}}\right)^{k-\ell}
\end{aligned}
$$




$$
\begin{aligned}
& =\frac{E_{7}}{E_{1}} \sum_{\ell=1}^{\infty} \sum_{k=\ell}^{\infty} a(2 j, \ell) m(4 \ell-1, k+\ell-1)\left(q \frac{E_{7}^{4}}{E_{1}^{4}}\right)^{k-1} \\
& =\sum_{k=1}^{\infty} \sum_{\ell=1}^{\infty} a(2 j, \ell) m(4 \ell-1, k+\ell-1) q^{k-1}\left(\frac{E_{7}}{E_{1}}\right)^{4 k-3} \\
& =\sum_{k=1}^{\infty} a(2 j+1, k) q^{k-1}\left(\frac{E_{7}}{E_{1}}\right)^{4 k-3} .
\end{aligned}
$$

Hence (3.2) holds for $j+1$.

For a positive integer $n$, we denote by $\pi_{7}(n)$ the highest power of 7 dividing $n$, and define $\pi_{7}(0):=\infty$. To prove Theorem 1.1, we need the following lemma.

Lemma $3.3-$ (Lemma 5.1, [13]). For all $i, j \geq 1$, we have

$$
\pi_{7}(m(i, j)) \geq\left\lfloor\frac{7 j-2 i-1}{4}\right\rfloor .
$$

We now establish a result on the 7-adic behavior of $a(j, k)$ that in combination with (3.3) implies Theorem 1.1.

Lemma 3.4 - For all $j, k \geq 1$, we have

$$
\pi_{7}(a(2 j, k)) \geq j+\left\lfloor\frac{7 k-7}{4}\right\rfloor
$$

and

$$
\pi_{7}(a(2 j+1, k)) \geq j+\left\lfloor\frac{7 k-7}{4}\right\rfloor
$$

PROOF : We may directly check that (3.7) holds for $j=1$; see Table 1 (notice that $a(2, k)=0$ when $k \geq 38$ ).

Table 1: 7-Adic evaluation of $a(2, k)$

\begin{tabular}{cccccccccccccc}
\hline \hline$k$ & 1 & 2 & 3 & 4 & 5 & 6 & 7 & 8 & 9 & 10 & 11 & 12 & 13 \\
$\pi_{7}(a(2, k))$ & 1 & 2 & 5 & 7 & 8 & 10 & 12 & 14 & 15 & 20 & 22 & 22 & 24 \\
\hline$k$ & 14 & 15 & 16 & 17 & 18 & 19 & 20 & 21 & 22 & 23 & 24 & 25 & 26 \\
$\pi_{7}(a(2, k))$ & 26 & 28 & 29 & 33 & 35 & 36 & 38 & 40 & 42 & 43 & 47 & 49 & 50 \\
\hline$k$ & 27 & 28 & 29 & 30 & 31 & 32 & 33 & 34 & 35 & 36 & 37 & 38 & 39 \\
$\pi_{7}(a(2, k))$ & 52 & 54 & 56 & 57 & 61 & 63 & 64 & 66 & 68 & 70 & 70 & $\infty$ & $\infty$ \\
\hline \hline
\end{tabular}


Suppose that (3.7) is true for some $j \geq 1$. In view of (3.1) and (3.6), we deduce that

$$
\begin{aligned}
\pi_{7}(a(2 j+1, k)) & =\pi_{7}\left(\sum_{i=1}^{\infty} a(2 j, i) m(4 i-1, i+k-1)\right) \\
& \geq \min _{i \geq 1}\left\{\pi_{7}(a(2 j, i))+\pi_{7}(m(4 i-1, i+k-1))\right\} \\
& \geq \min _{i \geq 1}\left\{j+\left\lfloor\frac{7 i-7}{4}\right\rfloor+\left\lfloor\frac{7 k-i-6}{4}\right\rfloor\right\} \\
& \geq j+\left\lfloor\frac{7 k-7}{4}\right\rfloor .
\end{aligned}
$$

This implies that (3.8) holds for $j$. Similarly,

$$
\begin{aligned}
\pi_{7}(a(2 j+2, k)) & =\pi_{7}\left(\sum_{i=1}^{\infty} a(2 j+1, i) m(4 i-3, i+k-1)\right) \\
& \geq \min _{i \geq 1}\left\{\pi_{7}(a(2 j+1, i))+\pi_{7}(m(4 i-3, i+k-1))\right\} \\
& \geq \min _{i \geq 1}\left\{j+\left\lfloor\frac{7 i-7}{4}\right\rfloor+\left\lfloor\frac{7 k-i-2}{4}\right\rfloor\right\} \\
& \geq j+\left\lfloor\frac{7 k-3}{4}\right\rfloor \\
& =j+1+\left\lfloor\frac{7 k-7}{4}\right\rfloor .
\end{aligned}
$$

This proves that (3.7) holds for $j+1$.

\section{OTHER CONGRUENCES}

Proof of TheOrem 1.2 : From (3.5) we know that

$$
\sum_{n=0}^{\infty} T_{7}(7 n+1) q^{n} \equiv 3 \frac{E_{7}}{E_{1}}=3 \sum_{n=0}^{\infty} b_{7}(n) q^{n} \quad(\bmod 7)
$$

from which we readily obtain (1.2). 
Proof of Theorem 1.4 : By (2.1), we find that

$$
\begin{aligned}
\sum_{n=0}^{\infty} T_{7}(n) q^{n}= & \frac{E_{7}^{3}}{E_{1}^{3}} \\
\equiv & E_{1}^{18}=E_{25}^{18}\left(\frac{1}{R}-q-q^{2} R\right)^{18} \\
\equiv & \frac{E_{25}^{18}}{R^{18}}\left(1+3 q R+2 q^{2} R^{2}+q^{3} R^{3}+2 q^{4} R^{4}+6 q^{5} R^{5}+2 q^{6} R^{6}+2 q^{7} R^{7}\right. \\
& +2 q^{8} R^{8}+3 q^{9} R^{9}+5 q^{10} R^{10}+3 q^{11} R^{11}+2 q^{12} R^{12}+3 q^{13} R^{13} \\
& +5 q^{14} R^{14}+2 q^{15} R^{15}+5 q^{16} R^{16}+6 q^{17} R^{17}+5 q^{18} R^{18}+q^{19} R^{19} \\
& +5 q^{20} R^{20}+5 q^{21} R^{21}+5 q^{22} R^{22}+4 q^{23} R^{23}+2 q^{24} R^{24}+4 q^{25} R^{25} \\
& +5 q^{26} R^{26}+4 q^{27} R^{27}+2 q^{28} R^{28}+5 q^{29} R^{29}+2 q^{30} R^{30}+q^{31} R^{31} \\
& \left.+2 q^{32} R^{32}+6 q^{33} R^{33}+2 q^{34} R^{34}+4 q^{35} R^{35}+q^{36} R^{36}\right) \quad(\bmod 7)
\end{aligned}
$$

With the help of (2.2), we get

$$
\begin{aligned}
\sum_{n=0}^{\infty} T_{7}(5 n+3) q^{5 n} & \equiv E_{25}^{18}\left(\frac{1}{R^{15}}+\frac{2 q^{5}}{R^{10}}+\frac{3 q^{10}}{R^{5}}+5 q^{15}+4 q^{20} R^{5}+2 q^{25} R^{10}+6 q^{30} R^{15}\right) \\
& \equiv E_{25}^{18}\left(\left(\frac{1}{R^{5}}-11 q^{5}-q^{10} R^{5}\right)^{3}+3 q^{15}\right) \\
& =E_{25}^{18}\left(\left(\frac{E_{5}^{6}}{E_{25}^{6}}\right)^{3}+3 q^{15}\right) \\
& \equiv E_{5}^{18}+3 q^{15} E_{25}^{18}(\bmod 7) .
\end{aligned}
$$

It follows that

$$
\begin{aligned}
\sum_{n=0}^{\infty} T_{7}(5 n+3) q^{n} & \equiv E_{1}^{18}+3 q^{3} E_{5}^{18} \equiv \frac{E_{7}^{3}}{E_{1}^{3}}+3 q^{3} \frac{E_{35}^{3}}{E_{5}^{3}} \\
& =\sum_{n=0}^{\infty} T_{7}(n) q^{n}+3 \sum_{n=0}^{\infty} T_{7}(n) q^{5 n+3} \quad(\bmod 7)
\end{aligned}
$$

which implies (1.3).

Replacing $n$ with $5 n+3$ in (1.3) yields

$$
\begin{aligned}
T_{7}\left(5^{3} n+\frac{3 \times 5^{3}-3}{4}\right) & \equiv T_{7}(25 n+18)+3 T_{7}(5 n+3) \\
& \equiv 4 T_{7}(5 n+3)+3 T_{7}(n) \quad(\bmod 7)
\end{aligned}
$$


and replacing $n$ with $5 n+3$ again and using (1.3) gives

$$
T_{7}\left(5^{4} n+\frac{3 \times 5^{4}-3}{4}\right) \equiv 5 T_{7}(n) \quad(\bmod 7)
$$

which is the $\alpha=1$ case of (1.4). Our result now follows easily by induction.

Comparing the coefficients of $q^{5 n+r}$ on both sides of (4.1) for $r \in\{0,1,2,4\}$, we obtain the following result.

Corollary 4.1 - If $r \in\{0,1,2,4\}$, then for all $n \geq 0$, we have

$$
T_{7}(25 n+5 r+3) \equiv T_{7}(5 n+r) \quad(\bmod 7)
$$

ProOF OF THEOREM 1.5 : Form (2.6), we find that for any $k \geq 0$,

$$
\begin{aligned}
& H_{13}\left(\eta^{k+13}\right)-13 H_{13}\left(\eta^{k+12}\right)+13(S+7) H_{13}\left(\eta^{k+11}\right)-13^{2}(S+3) H_{13}\left(\eta^{k+10}\right) \\
& +13\left(6 S^{2}+91 S+195\right) H_{13}\left(\eta^{k+9}\right)-13\left(74 S^{2}+481 S+845\right) H_{13}\left(\eta^{k+8}\right) \\
& +13\left(20 S^{3}+494 S^{2}+13^{3} S+3211\right) H_{13}\left(\eta^{k+7}\right) \\
& -13\left(222 S^{3}+2392 S^{2}+8619 S+10985\right) H_{13}\left(\eta^{k+6}\right) \\
& +13\left(38 S^{4}+1326 S^{3}+9464 S^{2}+13^{4} S+32955\right) H_{13}\left(\eta^{k+5}\right) \\
& -13\left(346 S^{4}+5486 S^{3}+31096 S^{2}+81289 S+85683\right) H_{13}\left(\eta^{k+4}\right) \\
& +13\left(36 S^{5}+1638 S^{4}+17238 S^{3}+83486 S^{2}+199927 S+199927\right) H_{13}\left(\eta^{k+3}\right) \\
& -13\left(204 S^{5}+4498 S^{4}+37518 S^{3}+162578 S^{2}+13^{5} S+13^{5}\right) H_{13}\left(\eta^{k+2}\right) \\
& +13\left(11 S^{6}+468 S^{5}+6422 S^{4}+43940 S^{3}+171366 S^{2}+13^{5} S+13^{5}\right) H_{13}\left(\eta^{k+1}\right) \\
& -S^{7} H_{13}\left(\eta^{k}\right)=0 .
\end{aligned}
$$

Using (2.5) and (4.3), we compute

$$
\begin{aligned}
H_{13}\left(\eta^{13}\right)= & S^{7}+51 \times 13^{2} S^{6}+808 \times 13^{2} S^{5}+398 \times 13^{3} S^{4}+110 \times 13^{4} S^{3} \\
& +16 \times 13^{5} S^{2}+13^{6} S \\
H_{13}\left(\eta^{14}\right)= & -506 S^{7}-13^{6}, \\
H_{13}\left(\eta^{15}\right)= & -15 S^{8}-38715 S^{7}-3410 \times 13^{2} S^{6}-25050 \times 13^{2} S^{5}-8310 \times 13^{3} S^{4}
\end{aligned}
$$




$$
\begin{aligned}
& -1660 \times 13^{4} S^{3}-15 \times 13^{6} S^{2}-15 \times 13^{6} S-13^{7}, \\
H_{13}\left(\eta^{16}\right)= & 530 S^{8}-13^{7}, \\
H_{13}\left(\eta^{17}\right)= & 119 S^{9}+7939 \times 13 S^{8}+131852 \times 13 S^{7}+7820 \times 13^{3} S^{6} \\
& +47022 \times 13^{3} S^{5}+1088 \times 13^{5} S^{4}+2839 \times 13^{5} S^{3} \\
& +391 \times 13^{6} S^{2}+34 \times 13^{7} S+13^{8} .
\end{aligned}
$$

Notice that

$$
\sum_{n=0}^{\infty} T_{7}(n) q^{n+4} \equiv q^{4} E_{1}^{18}=q^{130} E_{169}^{18} \eta^{18} \quad(\bmod 7)
$$

Applying the operator $H_{13}$ to both sides of (4.5) and then invoking (2.5), (4.4) and the $k=5$ case of (4.3), we obtain

$$
\begin{aligned}
\sum_{n=0}^{\infty} T_{7}(13 n+9) q^{13 n+13} & \equiv q^{130} E_{169}^{18} H_{13}\left(\eta^{18}\right) \\
& =q^{130} E_{169}^{18}\left(-27710 S^{9}-13^{8}\right) \quad(\bmod 7) .
\end{aligned}
$$

It follows that

$$
\begin{aligned}
\sum_{n=0}^{\infty} T_{7}(13 n+9) q^{n} & \equiv-27710 E_{1}^{18}-13^{8} q^{9} E_{13}^{18} \equiv 3 \frac{E_{7}^{3}}{E_{1}^{3}}+6 q^{9} \frac{E_{91}^{3}}{E_{13}^{3}} \\
& =3 \sum_{n=0}^{\infty} T_{7}(n) q^{n}+6 \sum_{n=0}^{\infty} T_{7}(n) q^{13 n+9} \quad(\bmod 7)
\end{aligned}
$$

which yields (1.5). Finally, (1.6) can be deduced from (1.5) in the same way that we deduced (1.4) from (1.3) above.

Similar to Corollary 4.1, one may obtain the following result from (4.6).

Corollary 4.3 - If $0 \leq s \leq 12$ and $s \neq 9$, then for all $n \geq 0$, we have

$$
T_{7}(169 n+13 s+9) \equiv 3 T_{7}(13 n+s) \quad(\bmod 7)
$$

Further, combining (1.4) and (1.6) with the fact that $T_{7}(20)=302022 \equiv 0(\bmod 7)$, we obtain two non-standard congruences for $T_{7}(n)$.

Corollary 4.3 - For all $\alpha \geq 0$, we have

$$
T_{7}\left(\frac{83 \times 5^{4 \alpha}-3}{4}\right) \equiv 0 \quad(\bmod 7)
$$


and

$$
T_{7}\left(\frac{83 \times 13^{4 \alpha}-3}{4}\right) \equiv 0 \quad(\bmod 7)
$$

We conclude this section by proving the following result, which includes Theorem 1.6 as the special case where $\ell=7$ and $k=1$.

Proposition 4.4 - Let $\ell$ and $k$ be integers satisfying $\ell \equiv k \equiv \lambda(\bmod 3)$ with $\lambda \in\{1,2\}$. Define $t(n)$ for $n \geq 0$ by

$$
\sum_{n=0}^{\infty} t(n) q^{n}=\frac{\left(q^{\ell} ; q^{\ell}\right)_{\infty}^{3}}{\left(q^{k} ; q^{k}\right)_{\infty}^{3}}
$$

Then

$$
t(3 n-\lambda) \equiv 0 \quad(\bmod 9)
$$

for all $n \geq 1$.

ProOF : It is easy to see that

$$
\frac{\left(q^{\ell} ; q^{\ell}\right)_{\infty}^{3}}{\left(q^{k} ; q^{k}\right)_{\infty}^{3}} \equiv \frac{\left(q^{\ell} ; q^{\ell}\right)_{\infty}^{3}\left(q^{k} ; q^{k}\right)_{\infty}^{6}}{\left(q^{3 k} ; q^{3 k}\right)_{\infty}^{3}} \quad(\bmod 9)
$$

Hence it suffices to show that $\left(q^{\ell} ; q^{\ell}\right)_{\infty}^{3}\left(q^{k} ; q^{k}\right)_{\infty}^{6}$ contains no term of the form $q^{3 n-\lambda}$ modulo 9 . Recall that the Jacobi's triple product identity [6, p. 14, Theorem 1.3.9] tells us

$$
(q ; q)_{\infty}^{3}=\sum_{n=0}^{\infty}(-1)^{n}(2 n+1) q^{n(n+1) / 2} .
$$

We may write

$$
(q ; q)_{\infty}^{3}=J_{0}+3 J_{1}
$$

where $J_{0}, J_{1} \in \mathbb{Z}[[q]]$ and the exponent of $q$ in every term of $J_{0}$ (resp. $J_{1}$ ) is congruent to 0 (resp. 1 ) modulo 3 . Hence given any positive integer $m$ satisfying $m \equiv \lambda_{m}(\bmod 3)$ with $\lambda_{m} \in\{1,2\}$, we have

$$
q m^{3}=J_{m, 0}+3 J_{m, \lambda_{m}}
$$

where $J_{m, 0}$ (resp. $3 J_{m, \lambda_{m}}$ ) consists of those terms in which the exponent of $q$ is 0 (resp. $\lambda_{m}$ ) modulo 3. 
Since $\ell$ and $k$ satisfy $\ell \equiv k \equiv \lambda(\bmod 3)$, we deduce that

$$
\left(q^{\ell} ; q^{\ell}\right)_{\infty}^{3}=J_{\ell, 0}+3 J_{\ell, \lambda}
$$

and

$$
\left(q^{k} ; q^{k}\right)_{\infty}^{3}=J_{k, 0}+3 J_{k, \lambda},
$$

and hence

$$
\left(q^{\ell} ; q^{\ell}\right)_{\infty}^{3}\left(q^{k} ; q^{k}\right)_{\infty}^{6} \equiv J_{\ell, 0} J_{k, 0}^{2}+3 J_{\ell, \lambda} J_{k, 0}^{2}+6 J_{\ell, 0} J_{k, 0} J_{k, \lambda} \quad(\bmod 9)
$$

One readily observes that there are no terms on the right in which the power of $q$ is congruent to $-\lambda$ modulo 3.

\section{FinAL REMARKS}

With the aid of modular forms, Atkin established a powerful result (see [4, Theorem 1.1]) on congruences for $k$-colored partitions, whose generating function is given by

$$
\sum_{n=0}^{\infty} p_{-k}(n) q^{n}=\frac{1}{(q ; q)_{\infty}^{k}}
$$

It would be interesting to investigate whether a similar result holds for the 7-regular analog of $p_{-k}(n)$ defined by

$$
\sum_{n=0}^{\infty} T_{7, k}(n) q^{n}=\frac{\left(q^{7} ; q^{7}\right)_{\infty}^{k}}{(q ; q)_{\infty}^{k}}
$$

\section{ACKNOWLEDGEMENT}

The authors would like to thank the referee for his/her careful reading and helpful comments on an earlier version of this paper. The second author was supported by the National Natural Science Foundation of China (No. 11501061) and the Fundamental Research Funds for the Central Universities (No. 2018CDXYST0024). The third author was supported by the National Natural Science Foundation of China (No. 11571143) and Jiangsu National Funds for Distinguished Young Scientists (No. BK20180044). 


\section{REFERENCES}

1. C. Adiga and D. Ranganatha, Congruences for 7 and 49-regular partitions modulo powers of 7 , Ramanujan J., 46(3) (2018), 821-833.

2. Z. Ahmed and N. D. Baruah, New congruences for $\ell$-regular partitions for $\ell \in\{5,6,7,49\}$, Ramanujan J., 40(3) (2016), 649-668.

3. G. E. Andrews, The theory of partitions, Reprint of the 1976 original. Cambridge Mathematical Library. Cambridge University Press, Cambridge, 1998. xvi+255 pp.

4. A. O. L. Atkin, Ramanujan congruences for $p_{-k}(n)$, Can. J. Math., 20 (1968), 67-78.

5. N. D. Baruah and K. Das, Parity results for 7-regular and 23-regular partitions, Int. J. Number Theory, 11(7) (2015), 2221-2238.

6. B. C. Berndt, Number theory in the spirit of Ramanujan, Student Mathematical Library, 34, American Mathematical Society, Providence, RI, 2006. xx+187 pp.

7. E. Boll and D. Penniston, The 7-regular and 13-regular partition functions modulo 3, Bull. Aust. Math. Soc. 93(3) (2016), 410-419.

8. R. Carlson and J. J. Webb, Infinite families of infinite families of congruences for $k$-regular partitions, Ramanujan J., 33(3) (2014), 329-337.

9. S. Cooper, The quintuple product identity, Int. J. Number Theory 2(1) (2006), 115-161.

10. S. Cui and N. S. S. Gu, Arithmetic properties of $\ell$-regular partitions, Adv. Appl. Math., 51(4) (2013), 507-523.

11. S. Cui and N. S. S. Gu, Congruences for 9-regular partitions modulo 3, Ramanujan J., 38(3) (2015), 503-512.

12. B. Dandurand and D. Penniston, $\ell$-divisibility of $\ell$-regular partition functions, Ramanujan J., 19(1) (2009), 63-70.

13. F. G. Garvan, A simple proof of Watson's partition congruences for powers of 7, J. Austral. Math. Soc. Ser. A, 36(3) (1984), 316-334.

14. M. D. Hirschhorn, An identity of Ramanujan, and applications, in: $q$-series from a contemporary perspective (South Hadley, MA, 1998), 229-234, Contemp. Math., 254, Amer. Math. Soc., Providence, RI, 2000.

15. M. D. Hirschhorn, The Power of $q$. A personal journey, Developments in Mathematics, 49. Springer, Cham, 2017. xxii+415 pp.

16. M. D. Hirschhorn, Ramanujan's tau function, in: Analytic number theory, modular forms and q-hypergeometric series, 311-328, Springer Proc. Math. Stat., 221, Springer, Cham, 2017. 
17. M. D. Hirschhorn and J. A. Sellers, Elementary proofs of parity results for 5-regular partitions, Bull. Aust. Math. Soc., 81(1) (2010), 58-63.

18. H. Jin and L. Zhang, Ramanujan-type congruences for $\ell$-regular partitions modulo 3, 5, 11 and 13, Int. J. Number Theory, 13(8) (2017), 1995-2006.

19. W, J. Keith, Congruences for 9-regular partitions modulo 3, Ramanujan J., 35(1) (2014), 157-164.

20. D. Penniston, The $p^{a}$-regular partition function modulo $p^{j}, J$. Number Theory, 94(2) (2002), 320-325.

21. D. Penniston, Arithmetic of $l$-regular partition functions, Int. J. Number Theory, 4(2) (2008), 295-232.

22. D. Ranganatha, Ramanujan-type congruences modulo powers of 5 and 7, Indian J. Pure Appl. Math., 48 (2017), 449-465.

23. N. Saikia and C. Boruah, Congruences of $\ell$-regular partition triples for $\ell \in\{2,3,4,5\}$, Acta Math. Vietnam, 42(3) (2017), 551-561.

24. L. Wang, Congruences for 5-regular partitions modulo powers of 5, Ramanujan J., 44(2) (2017), 343358.

25. L. Wang, Congruences modulo powers of 11 for some partition functions, Proc. Amer. Math. Soc., 146(4) (2018), 1515-1528.

26. L. Wang, Arithmetic properties of 7-regular partitions, Ramanujan J., 47 (2018), 99-115.

27. G. N. Watson, Ramanujans Vermutung über Zerfällungsanzahlen, J. Reine Angew. Math., 179 (1938), 97-128.

28. J. J. Webb, Arithmetic of the 13-regular partition function modulo 3, Ramanujan J., 25(1) (2011), 49-56.

29. E. X. W. Xia, Congruences for some $l$-regular partitions modulo $l$, J. Number Theory, 152 (2015), 105117.

30. E. X. W. Xia and O. X. M. Yao, Parity results for 9-regular partitions, Ramanujan J., 34(1) (2014), 109-117.

31. E. X. W. Xia and O. X. M. Yao, A proof of Keith's conjecture for 9-regular partitions modulo 3, Int. J. Number Theory, 10(3) (2014), 669-674. 